\title{
Themes in Human Work Interaction Design
}

\author{
Rikke Orngreen ${ }^{1}$, Annelise Mark Pejtersen ${ }^{2}$, Torkil Clemmensen ${ }^{3}$ \\ ${ }^{1}$ Center for Applied ICT, CBS, Denmark, orngreen@cbs.dk \\ ${ }^{2}$ Chair of IFIP TC 13, Denmark, ampcse@ mail.dk \\ ${ }^{3}$ Department of Informatics, CBS, Denmark, tc.inf@cbs.dk
}

\begin{abstract}
This paper raises themes that are seen as some of the challenges facing the emerging practice and research field of Human Work Interaction Design. The paper has its offset in the discussions and writings that have been dominant within the IFIP Working Group on Human Work Interaction Design (name HWID) through the last two and half years since the commencement of this Working Group. The paper thus provides an introduction to the theory and empirical evidence that lie behind the combination of empirical work studies and interaction design. It also recommends key topics for future research in Human Work Interaction Design.
\end{abstract}

Keywords: work analysis, interaction design, HCI tools, new ways of working

\section{Introduction - Scope and Research Area}

Technology is changing human life and work contexts in numerous ways: interfaces between collaborating individuals in advanced ICT networks, small and large-scale distributed systems, multimedia and embedded technologies, mobile technologies, and advanced "intelligent" robots. With this change towards new ways of working, an intensive demand has taken place for techniques and technologies that address contemporary issues related to communication, collaboration, learning, problem solving and information seeking in large information spaces of great variability. To address this comprehensive problem, an IFIP working group on Human Work Interaction Design (HWID) was established. Its expressed purpose was to reach a better understanding of the new challenges entailed in the design of technological support for modern, dynamic and complex work environments through a discussion of the interrelation between Work Analysis and Interaction Design within the field of Human Computer Interaction .

The main problem addressed is how we can understand, conceptualize and design for the complex and emergent contexts in which human life and work are now embroiled. This issue calls for cross disciplinary, empirical and theoretical approaches that focus on Human - Work Interaction design. 
The main target of this paper is to draw attention to this problem by discussing recent research topics which address this problem using different approaches, and secondly, to point to problems which need to be investigated further. Hopefully, this will encourage more empirical studies and conceptualisations of the interaction among humans, their work and other variegated contexts and the technology used both within and across these contexts.

\section{Background}

HWID organises Annual Working Conferences with printed papers, discussions and varied forms of interactions and collaborations during two days of workshop activities. HWID'05, a Working Conference took place in Rome at INTERACT'05, the International Conference of Human Computer Interaction. The focus was on "Describing Users in Context". HWID'06 took place at the University of Maidera. The theme was "Synthesizing work analysis and design sketching", with a particular focus on how to read design sketches within different approaches. HWID'07 took place in Rio de Janeiro at INTERACT'07. The focus was on "Social, Organisational and Cultural aspects of Human-Work Interaction Design". The inspiration of this paper is based on fruitful dialogs during these HWID activities.

The subjects raised in this paper stem from the authors' analysis of papers written in conjunction with discussions that took place. These papers were written by researchers from around the world; the topics covered a variety of disciplines and theoretical approaches in human sciences: psychology, anthropology, sociology, information and media sciences; computer sciences and engineering.

Human-Work Interaction Design is a comprehensive approach in HCI, and in order to provide an easy understanding and to illustrate the coverage of this research topic, we developed the model in figure 1.

Figure 1 shows examples of the characteristics of humans and work domain contents and the interaction during their tasks and decision activities, individually or in collaboration. Analysis of users' work and life, as well as the design of computer-based information systems, has inspired the development of numerous theories, concepts, techniques and methods. Some have been widely adopted by practitioners; others are used mainly by researchers, and these are naturally part of Human-Work Interaction design research, and they will obviously influence the work and user analysis as well as the technology design. This explains the top box.

Environmental contexts, such as national, cultural, social and organizational factors, impact the way in which users interact with computers in their work and life to the same extent as the nature of the application domain, the tasks, and the users' skills and knowledge. The analysis and design of Human-Work Interaction will necessarily also include these contextual factors. As a consequence hereof, the bottom box of figure 1 . 


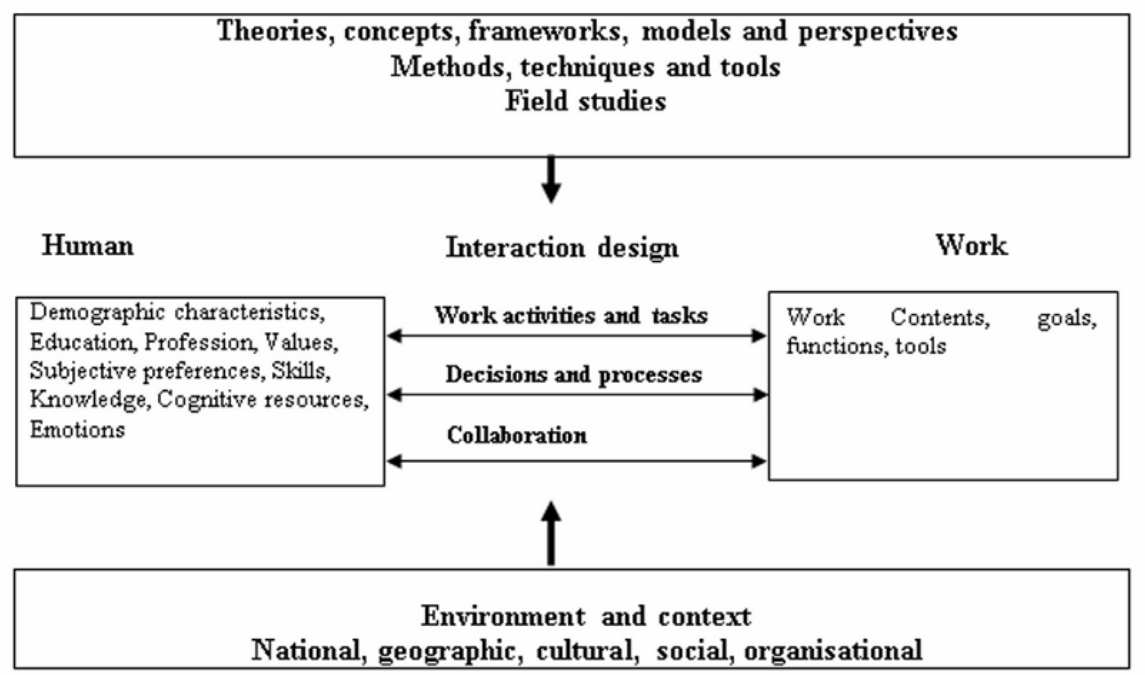

Figure 1 The Model of Human-Work Interaction Design

The analysis of the current HWID activities resulted in the identification of six main themes, which reflect those problems which the authors perceived to be of major concern in Human-Work Interaction Design:

Within Design processes

- Encouraging the dialogue between users and designers in the design process

- Bridging the HCI and Software Engineering gap by working with user requirements and collaboration in software development processes

- Supporting communication and design exploration through sketching

Within Work and User analysis

- Bridging the work analysis and interaction design gap through detailed case and field studies and action research projects empirical field studies.

- Rich contextual user descriptions, including methods to study unpredictable and opportunistic tasks

- Broadening the scope to Social, Organizational and Cultural aspects

Although this list presents these themes and problems as separated, they are of course intertwined and appear in different ways in many of the papers. Thus the following presentation of the papers within one of these headlines is of course exclusive for practical reasons, but as the reader will recognize, there are many overlapping themes and problems.

\section{Dialogue in the design process, between users and designers}

Design conceptualized as dialogue. Lopes 2006 provide a perspective on design as dialogue, consisting of a presentation of different definitions and 
different aspects of design, which could be argued as all being related to dialogue. Dialogue is considered in relation to objects, processes and disciplines of design. The author identifies some problems with the design-as-dialogue approach, mainly the complexity of the issue, and suggests a qualitative study that may help reveal ways to simplify and validate the approach.

Grounded theory to study users' responses. Nocera et al. 2005 suggest ways to support people's meetings and dialogues about their view of the world and their experience. They use grounded theory in the study of users' responses to an implementation of an ERP system in various countries; the authors investigate negotiation - as reconfiguration - between the roles of users and producers. The analysis shows very different attitudes toward the same systems when implemented in cultural diverse settings; it purports that making sense of the system in a particular work-context depends on cultural, organisational and individual preferences. These different attitudes and ways of use are particularly visible in breakdown situations; the authors argue for interaction between users and producers, and that producers should be able to observe and discuss users' breakdown situations, their frustration and workarounds.

Affinity Diagram for requirements elicitation. Bondarenko and Janssen 2005 use a different methodological approach. They use the Affinity Diagram method adapted from Hackos and Redish in the requirements elicitation process for the design of personal document management systems. Without losing the user's context and without requiring the reading of lengthy reports, this method helps structure large collections of mixed qualitative and quantitative data, and gives dynamic requirements (as opposed to static user profiles or task flows). However, the method as it is used per se results in abstraction of the requirements into a general level and hence results in difficulties in mapping the acquired results into system design.

Information acquisition using colleagues' verbal reports. Erlandsson and Jansson 2007. A new method for information acquisition called collegial verbalisation is explored using an empirical case study of vehicle operators being videotaped while driving a high-speed ferry, followed by some of their colleagues making verbal reports while watching this video data. These colleagues are very familiar with the driving task and the driver environment. The method is discussed in relation to the amount of information provided in general; the reliability of the data; and how it contributes to the detection of "buggy mental models" within the operators, and it is compared to more traditional forms of verbal reports. It is suggested that the method of collegial verbalisation may have combinatorial advantages that makes it more powerful as an analysis tool than the traditional forms of verbalisation, specifically if one wants to analyse work tasks that are dynamic and where the operators' behaviours are highly automated. However, more elaborate and systematic investigations must be conducted through experimental designs. 


\section{Bridging the HCI and Software Engineering gap}

User interface model and requirement tool. España et al. 2006 look at the gap between HCI and Software Engineering (SE); while SE is supposed to be strong in specifying functional requirements, $\mathrm{HCI}$ is centred on defining user interaction at the appropriate level of abstraction. An abstract model of the user interface represented by a ConcurTaskTrees model is used to enrich the functional specification, and a new tool called RETO that aims at requirement engineering is presented. The adoption of such a framework is promising and future empirical studies will show if the model can be justified.

Activity Theory and software development process. Software development is intrinsically a collaborative activity. Based on an analysis of current literature and software, Lewandowski and Bourguin 2006 find that current Software Development Environments seldom provide true integrated collaboration between developers, rather they offer only sharing of material or communication support, and do not support the actual work process of software development. Further, the ability to tailor the development environment is an issue, as features for allowing external applications to be nested into the environment are lacking. Grounding their work on Activity Theory, the authors describe how the eclipse (open source software) has been extended to accommodate for some of these deficiencies; it will be interesting to follow these features being implemented in future development processes.

User interface patternsin specific contexts. Stanard and Wampler 2005 focus on richness multi-dimensionality of user descriptions, and discuss how design patterns until now have been close to traditional usability guidelines; thus, there is a need to make design patterns to better support interaction of specific contexts. User Interface (UI) patterns are presented as a way of defining, applying and evaluating the translation of cognitive and collaborative requirements into meaningful human computer interaction in the designed interface, and then through this provide input to the development process. The described case involves an airport control system, and the discussion of command and control systems. The patterns are useful not only for the provision of training and inspiration to solutions, but also for the reuse of patterns that have been quality assured in complex and risk environments, such as command and control systems. The authors argue for the need for hierarchies of patterns that are based on a specific application-domain or work-domain to enhance the work-performance.

Work style modelling In the same vein, Campos and Nunes 2005, 2006 combine Work Style modelling with Usage-Centered Design with the objective of designing and evaluating better design tools. They describe the richness in the human-work interaction by using a new method of work style modelling, which has been applied to the work-context of interaction designers (as well as to collaborative software design). The work style is described from a set of informally defined values, and the set of styles which has been shown apparent in the work-context, are then more formally depicted and evaluated using diagrams 
and metrics. By modelling users' work style, the focus is put on work transitions (from one style of work to the other) and the designed solution ability to support the current context and changes in these - within the same application. The authors raise the question of whether it is possible to use work style modelling in other fields to describe flows between contexts of use.

\section{Sketching in Communication and design exploration}

Collaborative design process. Craft and Cairns 2006 offer experiences with sketching in a design process for an information visualization tool. The objective of the system is to support communication between users with different backgrounds - between biologists and mathematicians. The authors present an indepth analysis of the design process, showing that sketching as an integral part of a collaborative design process aids creativity, communication, and collaboration.

Representation of requirements based on Cognitive task analysis. Rozzi and Wong 2006 present a case study of how design sketching can be used as a technique for the representation of design requirements to help the creation of a common understanding between users, designers and software developers, during the development of a tool for supporting spatial-temporal reasoning in Air Traffic Control (ATC). The design process is based on a cognitive task analysis using the Critical Decision Method, relying on observation and video recordings as well as Contextual Inquiry interviews. The authors show how sketching was used to get insights into the design possibilities, but also find that spatial-temporal issues are difficult to illustrate with sketching techniques; thus, further work is needed.

Idea exploration and refinement of details. Orngreen 2006 reflects on what sketches are and on the use of design sketches when developing an e-learning platform for case-based learning. The author attempts to differentiate techniques that include sketches: rough hand drawn sketches - storyboards - prototypes, and how the emphasis changes from idea exploration to refinement of detail. The paper draws a distinction between a sketch as a design artefact that can stand alone and as part of a work process.

Reading design sketches using work analysis. Clemmensen 2006 investigates the role of design sketches in Interaction design and work analysis when designing a simple folder structure for e-learning software to be used for course administration at a higher education study programme. The author discusses how to conceptualize the process of reading design sketches using work analysis. The interface was evaluated using a think-aloud protocol, and was found to be less satisfactory than the earlier designs as it was 'long-winded'. This pointing to the need for future work on investigating the relation between the sketching techniques used and the design obtained in the development process.

Sketches to improve task performance. Although Pereira et al 2006 do not act in the space of IT, they adopt a human centred approach, illustrated with 
sketches, when looking to improve the performance of treadle pumps, to be used in developed countries. Similarly, Gaspar et al 2006, use annotations (words and sketches) to the photographs in their analysis and design studies when investigating ways to increase the amount of physical activity in the daily routine.

\section{Bridging the gap between work analysis and interaction design}

Cognitive work analysis and interface design. Upton and Doherty 2006 describe an approach to designing a visual application for a semiconductor manufacturing plant, which is seen as a complex, large-scale system requiring a structured design methodology. They present a design rationale supporting the explicit representation of hierarchies, the compatibility of views, and the use of contextual navigation. This design is derived from a cognitive work analysis, from which an Abstraction Decomposition Space (ADS) was made and the interface design was subsequently developed. The paper systematically describes the application of cognitive work analysis and the subsequent process of interface design, in an effort to bridge the design gap.

Future/vision seminars in action research. Based in user-centred and participatory design, Johansson and Sandblad 2006 investigate how a home care and help service organisation can be developed in order to be better prepared for future challenges. During their action research project, they used the future/vision seminar model, extended with assignments (such as: describe a day at work). The seminars resulted in the formulation of several scenarios, which again served as input to the design of a prototype.

Generic user interface for resource allocation. O'hargan and Guerlain 2006 provide a generic User Interface (UI) design for resource allocation problems. The UI is designed to support a person making resource allocation decisions (as opposed to purely automated decisions, often currently the case). They argue that their Resource Allocation Planning System (RAPS) can be adapted to several types of resource allocation domains. In future work it will be interesting to follow evaluations on whether or not it is capable of clearly supporting the work of people doing resource allocation.

Cognitive Task Analysis and Mapping analysis of team performance. Mapping analysis results into new designs in a multi-agent world. This is the focus of the proposal by McMorrow et al. 2005, who use cognitive task analysis to evaluate effective team performance in collaborative environments, such as air traffic management, in order to provide insights into how a technology becomes a 'team player'. A cognitive task analysis for effective team performance can help re-interpret the formal procedures often surrounding complex technological designs by negotiating among different perspectives and different meanings brought into the work environment. 
Cognitive Work Analysis and train driver interfaces. Jansson, Olsson and Erlandsson 2007 conducted field studies on the improvement of existing train driver interfaces within the framework of cognitive work analysis (CWA) (Rasmussen, Pejtersen, Goodstein 1994 together with the method for collegial verbalisation which produces think-aloud protocols from video-recordings. The analyses show that the driver works in three rather separate time intervals: a longrange, a short-term and an immediate sense perspective. The driver switches between these while travelling between two stations. A prototype of a planning area of a driver interface was developed, making these switches and feed-forward planning possible. Early tests using the user centred design approach show that the planning area of the interface supports the feed-forward decision strategy. However, the driver group also made substantial changes in the design, indicating that UCSD is an efficient tool in order to capture user competencies, and to bridge the gap between analysis and design.

\section{Rich contextual analysis of users}

Multidimensional, multimedia portraits of users. Recognizing the need for a general format for user descriptions, Orngreen et al. 2005 present a theoretical focus on human beings as they are perceived by the designers of the technologies of the 21st century. They argue that today software developers use techniques and methods in software development that embed mono-cultural and monodimensional models in various contexts which in the future must be replaced by rich portraits of human beings. In continuation thereof, the same group of authors in Nielsen et al. 2006 argues that cultural embeddings are significant in relation to HCI because the cultural context is also embedded in the methodological framework, the techniques and the tools that we apply. The authors suggest a research program that aims at developing a theoretical framework supporting the creation of rich multimedia portraits of the human user of multimodal technologies Orngreen et al. 2005; the authors point to a theory of complementary positions that insists on solid accounts from all observer positions in relation to perspective, standpoint and focus Nielsen et al. 2006.

Activity theory, situated action and distributed cognition models. The need for different positions is also a theme in Kimani et al. 2005 who use activity theory, situated action and distributed cognition models to study the nature of tasks in real world, natural settings. Within the context of mobile computing, they focus on how supplementary tasks, such as interacting with the device, are performed while the user does another primary task. Unpredictable and opportunistic tasks can be studied with these beyond task-centric approaches in order to provide rich and complex descriptions of users in the mobile domain. Information Science is another domain, which requires discussion of current approaches to model and describe empirically the different kinds of contexts. 
Information science Pejtersen et al. 2005 purport that we need not only an analysis of users' perceptual, cognitive, and social states, but also a deep understanding of how the users' contexts influence their interaction with artefacts such as a Digital Library. They propose that the problems raised within the information science field can provide a number of useful issues for discussion of the current approaches to describing users in context within the HCI field.

Critical Decision Method, Ethnography and Cognitive Work Analysis. Ham et al. 2005 present three case studies using three different methods, two for task-oriented design contexts (the Critical Decision Method and the Ethnography Method) and one for functional-oriented design contexts (the Cognitive Work Analysis Method, in particular the Abstraction Hierarchy). They argue that the critical decision method and the ethnography method provide useful and effective descriptions, enabling task-based design requirements in contexts of anticipated situations, while the abstraction hierarchy provides useful and effective descriptions in work domains of revolutionary designs for unanticipated situations. However, they miss an integrated method for obtaining information about user contexts, a method that is both task- and function-oriented.

The Activity Interview and Activity theory in HCI. Duignan, Noble and Biddle 2006 elaborate on their work on the activity interview based on cultural historical activity theory and in particular the activity checklist. The activity interview uses questions to get to an activity analysis as opposed to the abstract formulations of the activity checklist. The paper gives a thorough view of the activity theory relation to the HCI field and the activity list, and provides critical reflection of the list based on previous literature, as well as on personal experience. These discussions clearly bring forward issues for improvement at a very concrete level. In the future it will be interesting to follow the consequences that the activity interview has on design suggestions and how it can be seen in the resulting design. Further, it will be noteworthy to see whether the interview, as claimed, is appropriate for guiding the process of activity analysis, if performed by those who do not know activity theory or cultural historical activity theory.

\section{Impact of social, organizational, cultural and historical factors}

Avoiding cultural bias in usability tests. Clemmensen 2007 The CULTUSAB project is conducting an in-depth investigation of the key dimensions of culture that affect usability testing situations, including language, power distance, and cognitive style. All phases of the usability test are being evaluated for cultural impact, including planning, conducting, and reporting results. Special attention is being focused on subject-evaluator communication and cultural bias in the test design and structure of the user interface being tested. Experiments are being replicated in three countries: Denmark, India and China. The research will result 
in new testing methods and guidelines that increase the validity, by avoiding cultural bias, and allow for production of comparable results across countries.

Historical, national, and cultural factors in the work place. Rasmussen 2007 presents an empirical, qualitative study of Internet use in a National Film Archive in an Eastern European country. The purpose was to identify the use of and the attitude towards the Internet through field studies of individuals and organizations. The empirical study shows, that the staff at the archive only uses the Internet moderately in their work. It also shows that historical, national, and cultural factors can be used to explain the way people at work reacts to the new Internet technology. A cross-disciplinary study of the literature about Central and Eastern Europe made it possible to explain their behaviour and attitudes within a broader context.

A game based on cultural common sense. Anacleto Coutinho et al. 2007 argue that an effective educational process has to be instantiated in the local culture and that common sense knowledge represents culture. Common sense based games can be used to work on topics taught by teacher and can promote a meaningful learning, since the new knowledge (formal knowledge presented during classes) is related to pieces of knowledge already in the learners' cognitive structure (common sense knowledge). A common sense based game prototype to support the process of knowledge reinforcement of the content presented to students is presented. Teachers can set up a quiz game based on the Brazilian common sense knowledge. Preliminary analyses with users point out the potential for such approach.

\section{What did we learn?}

Obviously, a long list of specific and important problems can be derived from this research as described in each paper above. However, common issues are also addressed, which concern basic conditions of the HCI research.

While certain techniques and methods provide an integrated focus on analysis and design, most focus on either analysis or design. The strongest link between analysis and design is the general reliance on iteration as a way of developing products that fit the user needs and context, but within HWID other means and techniques have also been applied. Our papers and activities in the Working Group have operated on three levels:

- A field study level which involves an understanding of what actually goes on in a user environment.

- An applied level, which concentrates on methods and tools for analysis and design

- A theoretical level where academic disciplines have been selected to compensate for the shortcomings of single approaches when confronting the complexity of a design problem. 
While experimental design of prototypes is a necessary component of the iterative process of work studies, design and evaluation, consistent conceptualisations between work analysis and application evaluation are needed to provide results that are valid beyond discrete experiments, and can be generalised to other application domains and contexts. In some papers the authors present a satisfactory result of the application of a specific approach to solve their defined problem, few are not successful, but the majority of papers present approaches to their problems which the authors find promising, although still problematic, or yet unresolved, because no evaluation has taken place, or because it is unknown whether the approach can be generalized beyond the application domain.

The diverse combination of the approaches have mostly been driven by a particular work domain context, which is why the concluding discussion of theoretical concepts and tools applied in empirical work and prototype designs often refer to further research for validation of these in other application domain.

It is obvious that further work needs to be done in evaluating the designs that have been made, not only as they work in everyday practice, but also in relating them back to the insights that were gained from the initial work analysis and interaction design phases; in this way it is possible to better inform the concepts, methods and techniques applied.

Figure 2 shows the human actors who interact with work domains during their collaborative tasks and decision activities. A variety of application domains are studied in HWID research papers and the humans who perform this work also spans many different characteristics. Within HWID many means and techniques have been applied to study particular design problems, in most papers not one, but several theories, concepts, techniques and methods from several scientific disciplines have been necessary.

\begin{tabular}{|c|c|c|}
\hline \multicolumn{3}{|c|}{$\begin{array}{c}\text { Theories, concepts, frameworks, models and perspectives } \\
\text { Methods, techniques and tools } \\
\text { Field studies }\end{array}$} \\
\hline Human Actors & Interaction design & Work \\
\hline $\begin{array}{l}\text { Interaction designers } \\
\text { Course administrators } \\
\text { Designers, developers } \\
\text { Resource allocation staff } \\
\text { Biologists, Mathematicians }\end{array}$ & $\begin{array}{l}\text { Activities and tasks, Decisions, } \\
\text { Collaboration } \\
\text { Air traffic management } \\
\text { Education administration } \\
\text { Software engineering } \\
\text { Etc. }\end{array}$ & $\begin{array}{l}\text { Biology and Chemistry } \\
\text { Air Traffic Control } \\
\text { Case-based e-learning } \\
\text { Higher education } \\
\text { Software design } \\
\text { Airport control } \\
\text { Semiconductor manufacturing } \\
\text { Home care and help service } \\
\text { Resource allocation domains }\end{array}$ \\
\hline \multicolumn{3}{|c|}{$\begin{array}{l}\text { Environment and context } \\
\text { National, geographic, cultural, social, organisational }\end{array}$} \\
\hline
\end{tabular}

Figure 2 shows the application domains, the tasks and the users involved. The contextual factors in the buttom and the scientific approaches at the top. 
There are domains where the work analysis shows that ICT are not the obvious solution by adopting a human centred approach, illustrated with sketches, when looking to improve the performance of treadle pumps, to be used in developed countries (Pereira 2006). Similarly, Gaspar et al 2006, use annotations (words and sketches) to the photographs in their analysis and design studies when investigating ways to increase the amount of physical activity in the daily routine.

Necessary in a global world, but still very emergent area in HCI with few research papers submitted, is to provide a better understanding of the complex interplay between individual, social, organizational, cultural, historical and national factors during the use of technology now and in the future.

\section{Conclusion}

Finally, we need to mention that although we have introduced many and most, not all, of the hot issues from our papers contributed by participants at the IFIP HWID Working Conferences, this paper's contribution is mostly to give an up to the minute account of research approaches within Human-Work Interaction Design. The informed reader will recognize that apart from the focus on work studies, many of the problems and approaches presented in this sketch are common for other HCI activities, although based on a relative small amount of papers, generalizations are not possible.

In spite of this limitation, it is our hope that the variety of challenges presented in this paper will inspire other researchers and readers to participate and contribute to a better understanding of the complexity involved. We hope this paper promotes the use of knowledge, concepts, methods and techniques that enables work and user studies and design experiments to procure a better apprehension of the complex interplay between individual, social, cultural and organisational contexts.

\section{References}

Anacleto Coutinho, Ferreira, Pereira (2007) "Promoting Culture Sensitive Education through a Common Sense Based Game" in Mark-Pejtersen, Clemmensen et al (Eds.) (2007): Proceedings of the workshop: Social, Organisational and Cultural aspects of Human-Work Interaction Design, at the INTERACT 2007 conference, Rio, Brazil.

Bondarenko \& Janssen (2005). "Affinity diagram method: Bringing users' context into the system design", Proceedings of the workshop: Describing Users in Context Perspectives on Human-Work Interaction, the INTERACT 2005, Rome, Italy, p.34-37.

Campos \& Nunes (2005). "A human-work interaction design approach by modeling the user's work styles", Proceedings of the workshop: Describing Users in Context Perspectives on Human-Work Interaction, the INTERACT 2005, Rome, Italy, p.44-47.

Campos \& Nunes (2006): "Principles and Practice of Work Style Modeling: Sketching Design Tools", Clemmensen, T., Campos, P., Ørngreen, R., Pejtersen, A. M., \& Wong, 
W. (Eds.). (2006). Human work interaction design - designing for human work.: Springer, New York. p. 203-220

Clemmensen (2006): "A simple design for a complex work domain", Clemmensen, T., Campos, P., Ørngreen, R., Pejtersen, A. M., \& Wong, W. (Eds.). (2006). Human work interaction design - designing for human work.: Springer, New York. p. 221-240

Clemmensen (2007) "The Cultural Usability (CULTUSAB)" in Mark-Pejtersen, Clemmensen et al (Eds.) (2007): Proceedings of the workshop: Social, Organisational and Cultural aspects of Human-Work Interaction Design, at the INTERACT 2007 conference, Rio, Brazil.

Craft \& Cairns (2006): "Using Sketching to Aid the Collaborative Design of Information Visualisation Software", Clemmensen, T., Campos, P., Ørngreen, R., Pejtersen, A. M., \& Wong, W. (Eds.). (2006). Human work interaction design - designing for human work.: Springer, New York. p. 103-122

Duignan, Noble \& Biddle (2006): "Activity Theory for Design: From Checklist to Interview”, Clemmensen, Campos, Ørngreen, Pejtersen, \& Wong (Eds.). (2006). Human work interaction design - designing for human work.: Springer, N. Y.. p. 1-26

Erlandsson \& Jansson (2007) "Collegial verbalisation - a case study on a new method on information acquisition" in Mark-Pejtersen, Clemmensen et al (Eds.) (2007): Proceedings of the workshop: Social, Organisational and Cultural aspects of HumanWork Interaction Design, at the INTERACT 2007 conference, Rio, Brazil.

España, Pederiva, Ignacio Panach, Abrahão \& Pastor (2006): "Linking requirements specification with interaction design and implementation", Clemmensen, T., Campos, P., Ørngreen, R., Pejtersen, A. M., \& Wong, W. (Eds.). (2006). Human work interaction design - designing for human work.: Springer, New York. p. 123-134

Gaspar, Ventura, Pereira \& Santos (2006): “Continuous fitness at home: Designing exercise equipment for the daily routine" Clemmensen, T., Campos, P., Ørngreen, R., Pejtersen, A. M., \& Wong, W. (Eds.). (2006). Human work interaction design - designing for human work.: Springer, New York. p. 147-160

Ham, Wong \& Amaldi (2005). "Comparison of three methods for analyzing human work in terms of design approaches", Proceedings of the workshop: Describing Users in Context - Perspectives on Human-Work Interaction, the INTERACT 2005, Rome, Italy, p7-11.

Jansson, Olsson \& Erlandsson (2007): "Bridging the gap between analysis and design: Improving existing driver interfaces with tools from the framework of cognitive work analysis" in Mark-Pejtersen, Clemmensen et al (Eds.) (2007): Proceedings of the workshop: Social, Organisational and Cultural aspects of Human-Work Interaction Design, at the INTERACT 2007 conference, Rio, Brazil.

Johansson \& Sandblad (2006): "VIHO - Efficient IT Support in Home Care Services", Clemmensen, Campos, Ørngreen, Pejtersen, \& Wong, (Eds.). (2006). Human work interaction design - designing for human work.: Springer, New York. p. 47-66

Kimani, Gabrielli \& Catarci (2005)."Designing for primary tasks in mobile computing”, Proceedings of the workshop: Describing Users in Context - Perspectives on HumanWork Interaction, at the INTERACT 2005, Rome, Italy, p.11-17.

Lewandowski \& Bourguin (2006): "Improving collaboration support in software development activities" in Campos, Clemmensen, Orngreen, Wong, Mark-Pejtersen (2006) the Pre-conference proceedings of HWID'06. p. 33-45

Lopes (2006): "Design as Dialogue - a New Design Framework", Clemmensen, T., Campos, P., Ørngreen, R., Pejtersen, A. M., \& Wong, W. (Eds.). (2006). Human work interaction design - designing for human work.: Springer, New York. p. 241-250 
Mark-Pejtersen \& Fidel (2005). "A Multi-Dimensional Approach to Describing Digital Library Users in Context", Proceedings of the workshop: Describing Users in Context Perspectives on Human-Work Interaction, the INTERACT 2005, Rome, Italy, p.18-26.

Mark-Pejtersen, Clemmensen et al (2007): Proceedings of the workshop: Social, Organisational and Cultural aspects of Human-Work Interaction Design, at the INTERACT 2007 conference, Rio, Brazil.

McMorrow, Amaldi \& Boiardi (2005). "Approaches to designing for highly collaborative, distributed and safety-critical environments", Proceedings of the workshop: Describing Users in Context - Perspectives on Human-Work Interaction, the INTERACT 2005, Rome, Italy, p.38-43.

Nielsen, Yssing, Clemmensen, Orngreen, Nielsen, Levinsen (2006):" The human being in the 21st century- Design perspectives on the representation of users in IS" Clemmensen, Campos, Ørngreen, Pejtersen, A. M., \& Wong, W. (Eds.). (2006). Human work interaction design - designing for human work.: Springer, New York. p. 93-102

Nocera, Dunckley \& Hall (2005). "Reconfiguring producers and users through human-work interaction", Proceedings of the workshop: Describing Users in Context-Perspectives on Human-Work Interaction, at the INTERACT 2005, Rome, Italy, p.27-33.

O'Hargan \& Guerlain (2006): Design of a Ressource Allocation Planning System” Clemmensen, Campos, Ørngreen, Pejtersen, \& Wong, W. (Eds.). (2006). Human work interaction design - designing for human work.: Springer, New York. p. 67-92

Orngreen (2006): "The Design Sketching Process", Clemmensen, T., Campos, P., Ørngreen, R., Pejtersen, A. M., \& Wong, W. (Eds.). (2006). Human work interaction design - designing for human work.: Springer, New York. p. 185-202

Orngreen, Clemmensen, Nielsen, Christiansen, Levinsen, Nielsen \& Yssing (2005) "The Human Being in the $21^{\text {st }}$ Century -Design perspectives on the representation of users in IS development, Proceedings of the workshop: Describing Users in Contex - Perspectives on Human-Work Interaction, at the INTERACT 2005, Rome, Italy, p.54-59.

Pereira, Malca, Gaspar \& Ventura (2006): "Human Motion Analysis in Treadle Pump Devices”, Clemmensen, Campos, Ørngreen., Pejtersen, \& Wong (Eds.). (2006). Human work interaction design - designing for human work.: Springer, New York. p. 135-146

Rasmussen (2007), "Internet Use in Eastern Europe A Case Study" in Mark-Pejtersen, Clemmensen et al (Eds.) (2007): Proceedings of the workshop: Social, Organisational and Cultural aspects of Human-Work Interaction Design, at the INTERACT 2007 conference, Rio, Brazil.

Rasmussen, Jens, Pejtersen,A.M. and Goodstein, L.P. (1994): Cognitive Systems Engineering. JohnWiley, London.

Rozzi \& Wong (2006)" Design Sketching for Space and Time”, Clemmensen, T., Campos, P., Ørngreen, R., Pejtersen, A. M., \& Wong, W. (Eds.). (2006). Human work interaction design - designing for human work.: Springer, New York. p. 161-184

Stanard \& Wampler (2005). "Work-centered user interface patterns" Proceedings of the workshop: Describing Users in Context - Perspectives on Human-Work Interaction, at the INTERACT 2005, Rome, Italy, p.48-53.

Upton \& Doherty: "Visual Representation of Complex Information Structures in High Volume manufacturing", Clemmensen, T., Campos, P., Ørngreen, R., Pejtersen, A. M., \& Wong, W. (Eds.). (2006). Human work interaction design - designing for human work.: Springer, New York. p. 27-46 